\title{
LARGEST CLAIMS REINSURANCE (LCR). \\ A QUICK METHOD TO CALCULATE LCR-RISK RATES FROM EXCESS OF LOSS RISK RATES
}

\section{G. Benktander \\ Switzerland}

Let us denote by $E(x)$ the pure risk premium of an unlimited excess cover with the retention $x$ and by $H(x)$ and $m(x)$ the corresponding expected frequency and severity.

We thus have $E(x)=H(x) \cdot m(x)$.

$H(x)$ is a non-increasing function of $x$ and for practical purposes we can assume that it is decreasing; $H^{\prime}(x)<0$. The equation $H(x)=n$ has then only one solution $x_{n}$, where $n$ is a fixed integer.

Let $E_{n}$ denote the risk premium for a reinsurance covering the $n$ largest claims from the bottom.

Let us define $E_{n}^{\prime}=n x_{n}+E\left(x_{n}\right)=n\left(x_{n}+m\left(x_{n}\right)\right)$. Intuitively we feel that $E_{n}^{\prime}$ is a good approximation for $E_{n}$.

We shall first show that when the claims size distribution is Pareto and the number of claims is Poisson distributed, $E_{n}^{\prime}$ is a good approximation for $E_{n}$, being slightly on the safe side. We further include a proof given by G. Ottaviani that the inequality $E_{n}<E_{n}^{\prime}$ always holds.

In the Pareto case we have

$$
H(x)=t(\mathrm{I}-F(x))=t \cdot x^{x}
$$

where the Poisson parameter $t$ stands for the expected number of claims in excess of I (equal to a suitably chosen monetary unit) and

$$
m(x)=\frac{x}{\alpha-\mathrm{I}} .
$$

The retention $x_{n}$ over which we expect $n$ claims should satisfy

$$
n=H\left(x_{n}\right)=t \cdot x_{n}^{-\alpha}
$$

which gives

$$
t=n \cdot x_{n}^{\mathrm{x}}
$$

or

$$
x_{n}=\left(\frac{t}{n}\right)^{1 / \alpha}
$$


According to B. Berliner [2] we have, when the number of claims is Poisson distributed

$$
E_{n}=t^{1 / \alpha} \sum_{i=1}^{n} \frac{\mathrm{I}}{\Gamma(i)} \cdot \Gamma_{t}\left(i-\frac{\mathrm{I}}{\alpha}\right)
$$

where

$$
\Gamma_{t}(n)=\int_{0}^{t} e^{-u} \cdot u^{n-1} d u
$$

Replacing the incomplete Gamma function $\Gamma_{t}$ by $\Gamma_{\infty}=\Gamma$ we arrive at

$$
\bar{E}_{n}=t^{1 / \alpha} \cdot \frac{\alpha}{\alpha-\mathrm{I}} \cdot \frac{\mathrm{I}}{\Gamma(n)} \cdot \Gamma\left(n+\mathrm{I}-\frac{\mathrm{I}}{\alpha}\right)
$$

which formula was given by $\mathrm{H}$. Ammeter already in I964 [I]. Obviously $E_{n}<\bar{E}_{n}$.

In all cases when $t$ is large compared to $n$, we have

$$
\frac{E_{n}}{\bar{E}_{n}}(n, \alpha ; t) \text { very close to } \mathrm{I} \text {. }
$$

If in a practical situation $t$ is too small we can always increase $t$ by decreasing the monetary unit, in other words by enlarging to the left the range of the Pareto distribution.

Inserting $t=n x_{n}^{\alpha}$, as deduced above, in $\bar{E}_{n}$, we obtain

$$
\bar{E}_{n}=n^{1 / \alpha} \cdot x_{n} \cdot \frac{\alpha}{\alpha-\mathrm{I}} \cdot \frac{\mathrm{I}}{\Gamma(n)} \Gamma\left(n+\mathrm{I}-\frac{\mathrm{I}}{\alpha}\right) \text {. }
$$

\section{However}

$$
E_{n}^{\prime}=n\left(x_{n}+m\left(x_{n}\right)\right)=n \cdot x_{n} \cdot \frac{\alpha}{\alpha-\mathrm{I}}=x_{n} \cdot \frac{\alpha}{\alpha-\mathrm{I}} \frac{\Gamma(n+\mathrm{I})}{\Gamma(n)} .
$$

Thus we have

$$
\frac{\tilde{E}_{n}}{E_{n}^{\prime}}=\frac{n^{1 / \alpha} \cdot \Gamma\left(n+\mathrm{I}-\frac{\mathrm{I}}{\alpha}\right)}{\Gamma(n+\mathrm{I})}
$$


Tabulation of

$$
\begin{array}{rccc} 
& \multicolumn{4}{c}{\frac{\bar{E}_{n}}{E_{n}^{\prime}}} \\
& & \\
n & \alpha=2 & \alpha=2.5 & \alpha=3 \\
\text { I } & 0.886 & 0.894 & 0.903 \\
2 & 0.940 & 0.943 & 0.948 \\
3 & 0.959 & 0.96 \text { I } & 0.964 \\
4 & 0.969 & 0.97 \text { I } & 0.973 \\
5 & 0.975 & 0.976 & 0.978 \\
. & & & \\
. & & & \\
\text { IO } & 0.988 & 0.988 & 0.989
\end{array}
$$

The figures illustrate

that the approximation is good,

that the approximation is on the safe side, and that the approximation is rather invariant to variations of the parameter alpha within the given interval.

The safety margin in the approximation $-E_{n}^{\prime}$ replacing $\bar{E}_{n}$-is roughly of the form constant/n.

This is illustrated below for alpha $=2.5$

$\begin{array}{rcc}n & \frac{\bar{E}_{n}}{E_{n}^{\prime}} & n \cdot \frac{E_{n}^{\prime}-\bar{E}_{n}}{E_{n}^{\prime}} \\ \text { I } & 0.894 & 0.1 \mathrm{I} \\ 2 & 0.943 & 0.1 \mathrm{I} \\ 3 & 0.96 \mathrm{I} & 0.12 \\ 4 & 0.97 \mathrm{I} & 0.12 \\ 5 & 0.976 & 0.12 \\ \cdot & & \\ \cdot & & \\ \text { I0 } & 0.988 & 0.12\end{array}$

We have thus shown that in the Pareto case

$$
\frac{E_{n}}{E_{n}^{\prime}} \sim \mathrm{I}
$$

and

$$
\begin{aligned}
E_{n}<\bar{E}_{n}<E_{n}^{\prime}=n x_{n}+E\left(x_{n}\right) & =n x_{n}+n \frac{x_{n}}{\alpha-\mathrm{I}}= \\
& =n x_{n} \cdot \frac{\alpha}{\alpha-\mathrm{I}}=\alpha \cdot E\left(x_{n}\right) .
\end{aligned}
$$


Thus

$$
\frac{E_{n}}{E\left(x_{n}\right)} \sim \alpha
$$

This means that the LCR risk premium is approximately equal to alpha times the risk premium of an XL cover with a retention chosen in such a way that the expected number of claims is equal to the number of LCR-claims protected.

In the Poisson-Pareto case $E_{n}^{\prime}$ gives a handy and fairly good approximation of $\mathrm{E}_{n}$. The reader is invited to examine other claims size distributions $F(x)$ which are of importance in the practice.

Most such distributions will for all $x>x_{0}$ have $m^{\prime \prime}(x)<0$. We believe that $m^{\prime \prime}(x)<0$ will guarantee that $E_{n}^{\prime}$ will be a good approximation of $E_{n}$ with $E_{n}^{\prime}>E_{n}$.

We now give a proof by G. Ottaviani that the inequality $E_{n}<$ $E_{n}^{\prime}$ is valid for any $n$ and for arbitrary distribution functions of the number of claims and of the claim size.

We do not even need the condition of section 2 that the equation $H(x)=n$ has only one solution since the proof will be valid for any $X_{n}$, such that $H\left(x_{n}\right)=n$.

Let $s$ denote the total number of claims which occur and $N=$ $\min (s, n)$. We thus allow for the possibility that less than $n$ claims occur.

Let $X_{n}$ be the set consisting of the $N$ largest claims.

Let

$$
\begin{aligned}
& \vee\left(X_{n}\right)=E(N) \\
& \vee\left(X_{n}\right) \leq n
\end{aligned}
$$

Let $\mu\left(X_{n}\right)=E_{n} / \nu\left(X_{n}\right)$ be the expected value of a claim in the set $X_{n}$.

Analoguesly we denote by $X_{n}^{\prime}$ the set consisting of all claims exceeding $x_{n}$, the expected number of claims exceeding $x_{n}$ by $v\left(X_{n}^{\prime}\right)$ and the expected value of a claim in the set $X_{n}^{\prime}$ by $\mu\left(X_{n}^{\prime}\right)$.

We thus have

$$
\vee\left(X_{n}^{\prime}\right)=n
$$

and

Let

$$
\begin{aligned}
& \mu\left(X_{n}^{\prime}\right)=x_{n}+m\left(x_{n}\right) . \\
& Y_{n}=X_{n} \smile X_{n}^{\prime} \\
& Z_{n}=\left(X_{n} \smile X_{n}^{\prime}\right)-X_{n}^{\prime} \\
& Z_{n}^{\prime}=\left(X_{n} \smile X_{n}^{\prime}\right)-X_{n}
\end{aligned}
$$


$\nu\left(Y_{n}\right), \mu\left(Y_{n}\right), \nu\left(Z_{n}\right), \mu\left(Z_{n}\right), v\left(Z_{n}^{\prime}\right), \mu\left(Z_{n}^{\prime}\right)$ are defined analoguesly to $\nu\left(X_{n}\right)$ and $\mu\left(X_{n}\right)$. From the above definition it follows directly that

$$
\begin{gathered}
\mu\left(Z_{i}\right)<x_{n} \text { and } \\
\mu\left(Z_{i}^{\prime}\right) \geq x_{n} .
\end{gathered}
$$

Thus

$$
E_{n}=\nu\left(X_{i}\right) \cdot \mu\left(X_{i}\right)=\nu\left(Y_{i}\right) \mu\left(Y_{i}\right)+\nu\left(Z_{i}\right) \mu\left(Z_{i}\right)
$$

and

$$
E_{n}^{\prime}=\nu\left(X_{i}^{\prime}\right) \cdot \mu\left(X_{i}^{\prime}\right)=\nu\left(Y_{i}\right) \mu\left(Y_{i}\right)+\nu\left(Z_{i}^{\prime}\right) \mu\left(Z_{i}^{\prime}\right) .
$$

From (I) and (2) it follows that

$$
v\left(Y_{i}\right)+v\left(Z_{i}\right)=v\left(X_{i}\right) \leq n=v\left(X_{i}^{\prime}\right)=v\left(Y_{i}\right)+v\left(Z_{i}^{\prime}\right) .
$$

Thus

$$
v\left(Z_{i}\right) \leq v\left(Z_{i}^{\prime}\right)
$$

From (3) and (4) it follows that

$$
\mu\left(Z_{i}\right)<\mu\left(Z_{i}^{\prime}\right)
$$

and from (7) and (8)

$$
v\left(Z_{i}\right) \cdot \mu\left(Z_{i}\right)<v\left(Z_{i}^{\prime}\right) \mu\left(Z_{i}^{\prime}\right)
$$

Adding $\vee\left(Y_{i}\right) \cdot \mu\left(Y_{i}\right)$ to both sides of (9) and using (5) and (6) leads to

$$
E_{n}<E_{n}^{\prime} \quad \text { q.e.d. }
$$

\section{REFERENCES}

LI Ammeter H., The Rating of "Largest Claim" Reinsurance Covers. Quarterly Letter from the Algemeene Reinsurance Companies Jubilee Number 2, July I964.

[2] Berliner B., Correlations between Excess of Loss Reinsurance Covers and Reinsurance of the $\mathrm{n}$ Largest Claims. The ASTIN Bulletin Vol. VI, Part III, May 1972. 